\title{
Facts about Wildlife Diseases: Raccoon-Borne Pathogens of Importance to Humans-The Raccoon Roundworm ${ }^{1}$
}

\author{
Caitlin Jarvis and Mathieu Basille ${ }^{2}$
}

Northern raccoons (Procyon lotor, Figure 1) can carry many diseases that present significant health hazards to both people and pets. Some of these diseases are asymptomatic, showing no signs of infection, and often do not affect raccoons, but can still be passed on and deadly to other animals, including humans. Because it is not possible to be certain if a wild animal is sick, it is safer to consider the animal a hazard and avoid it. Contact animal control or a wildlife rehabilitator if you suspect an animal is sick or behaving abnormally (contact details for Florida wildlife rehabilitators can be found on the Florida Fish and Wildlife Conservation Commission website). Sick wild animals can act tame and confused but should never be approached as if they are domesticated. They are still wild animals that will likely see you as a threat, and can act aggressively. Due to their successful adaptation to urban environments, it is common for raccoons to come into contact with humans. This document is part of a series addressing health hazards associated with raccoons and specifically describes the raccoon roundworm, Baylisascaris procyonis, and the associated baylisascariasis disease. Information on raccoon-borne parasites and viruses and bacteria, can be found in other documents of this series. Baylisascariasis is a potentially deadly disease that is emerging in the United States and is the focus of this document.

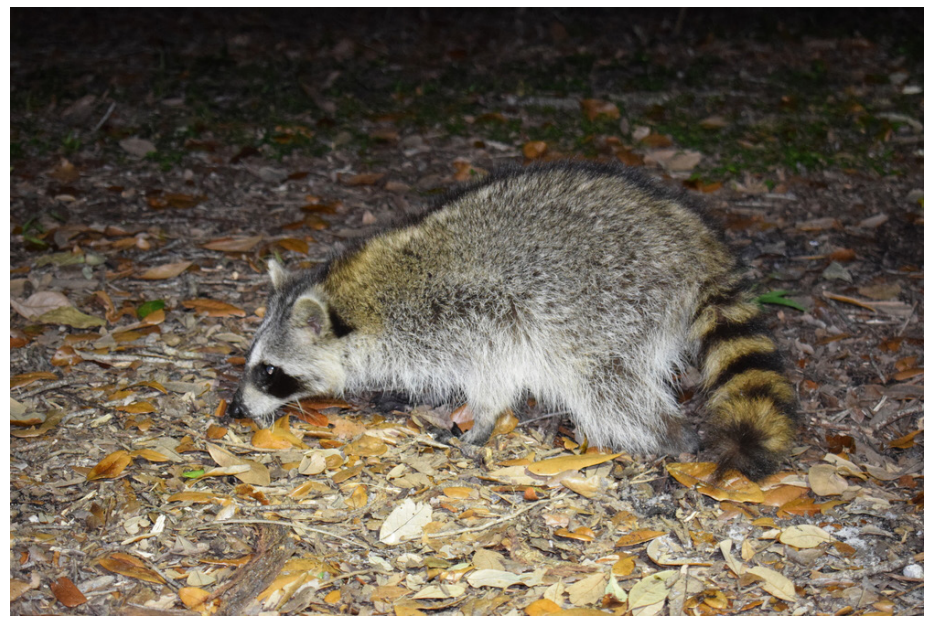

Figure 1. A young female raccoon in Broward County, south Florida. Credits: Mathieu Basille, UF/IFAS

\section{Baylisascariasis}

Baylisascariasis is caused by infection with the parasite Baylisascaris procyonis, a nematode in the family Ascarididae often called raccoon roundworm (Figure 2). As the name suggests, the parasite's primary host is the raccoon, but the parasite can also infect other animals, called paratenic hosts. A paratenic host is any animal a given parasite can infect but in which the parasite cannot develop into maturity and reproduce. Although the larvae cannot develop in a paratenic host, they are still able to migrate throughout the host's body, causing potentially debilitating

1. This document is WEC435, one of a series of the Department of Wildlife Ecology and Conservation, UF/IFAS Extension. Original publication date November 2020. Visit the EDIS website at https://edis.ifas.ufl.edu for the currently supported version of this publication.

2. Caitlin Jarvis, former technician, UF/IFAS Fort Lauderdale Research and Education Center; and Mathieu Basille, assistant professor, Department of Wildlife Ecology and Conservation, UF/IFAS Fort Lauderdale Research and Education Center, Davie, FL 33314.

The Institute of Food and Agricultural Sciences (IFAS) is an Equal Opportunity Institution authorized to provide research, educational information and other services

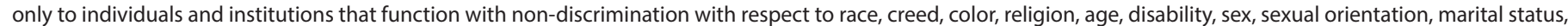

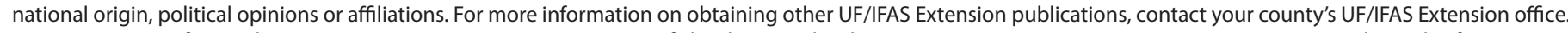
U.S. Department of Agriculture, UF/IFAS Extension Service, University of Florida, IFAS, Florida A \& M University Cooperative Extension Program, and Boards of County Commissioners Cooperating. Nick T. Place, dean for UF/IFAS Extension. 
and fatal disease. B. procyonis has one of the widest ranges of paratenic hosts known. Infected accoons can pass infective eggs to other animals, humans included. The eggs then hatch into larvae, which cause the illness. Unless the infection is very heavy, raccoons will probably show no symptoms of infection; avoid contact with raccoons and all wild animals, even if they seem healthy.

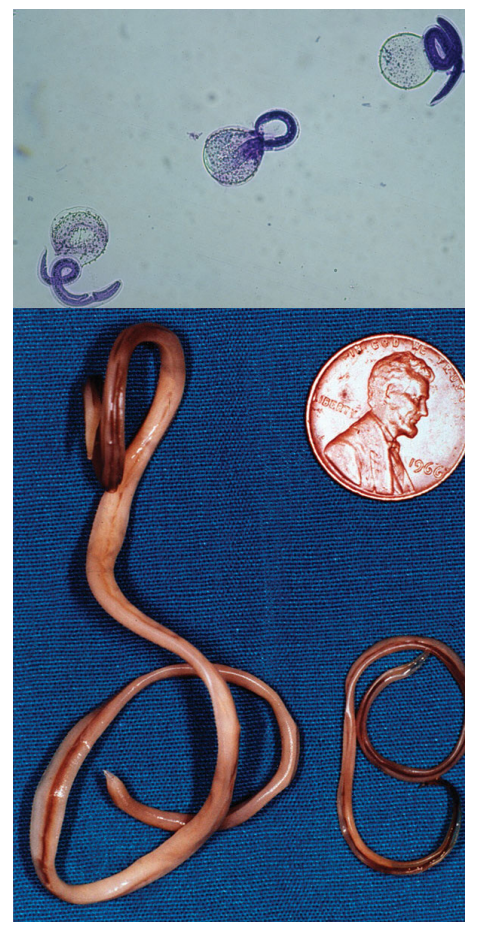

Figure 2. Larvae (top, magnification $\times 40$ ) and adults (bottom) Baylisacaris procyonis. Adult females (bottom left) are about $20-24 \mathrm{~cm}$ long; males (bottom right) are about 9-12 cm long.

Credits: Pictures from Shafir et al. 2011 and Roussere et al. 2003 (public domain)

B. procyonis infection occurs in three known clinical presentations, each named after the area of infection. Neural larva migrans (NLM), ocular larva migrans (OLM), and visceral larva migrans (VLM) occur when the nervous system, the eyes, and various other organs, respectively, are infected. Larva migrans occurs when immature larval worms migrate throughout the body of hosts. Other parasites, notably Toxocara spp., are able to cause larva migrans as well. It can be assumed in this document that unless otherwise stated, all cases of larva migrans mentioned are caused by B. procyonis.

\section{Raccoons, Humans, and Baylisascariasis}

Raccoons not only adapt to live near humans but in many cases benefit from urbanization. They readily make use of increased food availability from garbage cans, pet food, chicken coops, bird feeders, crops, and intentional feeding. Predators of raccoons have been greatly reduced, especially in highly urbanized areas. This leads to greater population densities and smaller territories with overlap, increasing promiscuity, and the risk of disease transmission between raccoons. Furthermore, raccoons use communal sites, known as latrines, to defecate, increasing the chance that people and pets will come into contact with contaminated feces.

B. procyonis reaches highest densities in raccoons in the Midwest, Northeast, and West Coast of North America (Figure 3). The Southeast United States was considered low risk, but recent cases of clinical and asymptomatic infection have emerged, including in Florida. Residents of these areas should be aware that $B$. procyonis has the potential to become more widespread in their area. Raccoons have been introduced in many areas worldwide for the fur trade and as pets, bringing $B$. procyonis with them.

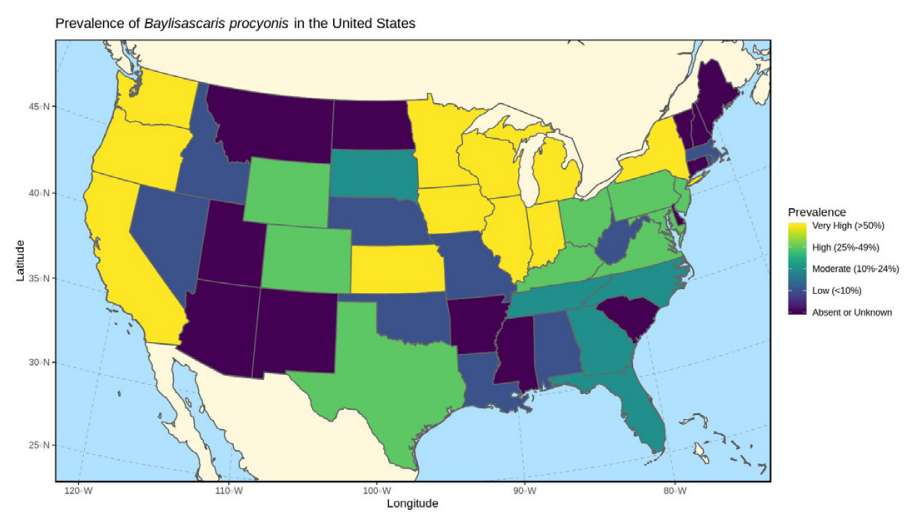

Figure 3. Prevalence of Baylisascaris procyonis in raccoons in the United States.

Credits: Map recreated from data from Sapp et al. 2016

All known cases of NLM have occurred in the United States and Canada. A small number of cases of ocular larva migrans have been documented in South America and Europe, likely from pet raccoons, other procyonids, or dogs.

\section{Life Cycle of Baylisascaris procyonis}

Adult B. procyonis are large, tan roundworms, females measuring $20-24 \mathrm{~cm}$, and males $9-12 \mathrm{~cm}$ in length. A single female worm can produce an estimated 115,000-179,000 eggs per day. It can take as few as 11 days to as long as several weeks for eggs shed in feces to become infective. Once infective, eggs can remain viable in the environment for years with adequate humidity. Even in dry conditions, they remain infective for many months and have been shown to withstand freezing at $-20^{\circ} \mathrm{C}\left(-4^{\circ} \mathrm{F}\right)$ for 8 weeks. When ingested by a paratenic host, the eggs hatch and migrate to the lungs, where they can then distribute throughout the body. In these hosts, the larvae are unable to mature, reaching a length of only $1.5-1.9 \mathrm{~mm}$. Most of these larvae 
will be encapsulated in granulomas, a mass of tissue and cells produced by the body in response to infection. These larvae continue to live for months but are not able to migrate further and typically cause few problems in larger animals. An estimated 5-7 percent of larvae, however, can migrate into the central nervous system. These larvae are the causative agent of clinical baylisascariasis. The number of larvae needed to cause disease is related to the size of the host. For example, a single larva in the brain of a mouse or bird can be fatal, while it takes several larvae to cause severe NLM in human hosts.

Young raccoons have a higher rate of infection than adults. Evidence has shown that raccoons can partially eliminate the parasite during winter in northern states. Whether this is due to the seasonal changes directly, or indirectly through changes in latrine use, rearing of young, or shifts in food sources is unknown. It is also unknown if this phenomenon is present in Florida. Raccoons become infected by ingesting infected prey or contaminated feces at raccoon latrines, or through contact with infected animals at other communal sites. Altered behavior of infected prey species makes them more susceptible to being eaten by a raccoon. The life cycle of B. procyonis is summarized in Figure 4.

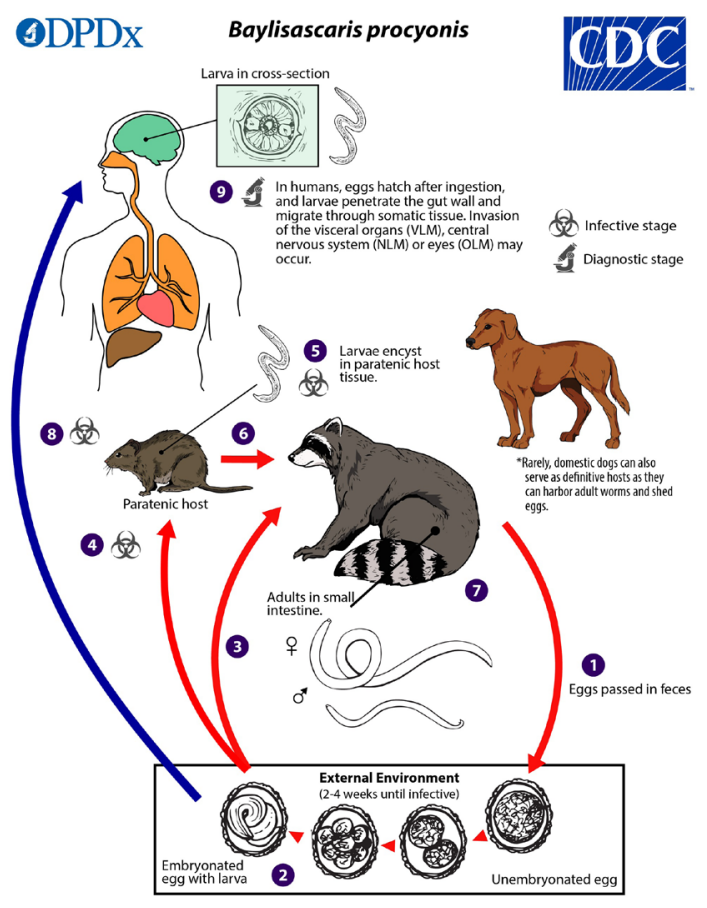

Figure 4. Life cycle of Baylisascaris procyonis.

Credits: Centers for Disease Control and Prevention. Public Domain

\section{Baylisascariasis in Humans}

From 1949 to 2017, 28 human cases of NLM, 24 cases of OLM, and one suspected case of VLM have been reported in the United States (Figure 5). Due to difficult diagnosis, it is likely that this is an underreported disease, especially before it was widely recognized in the medical field.

Baylisascariasis cases are currently increasing, either due to better detection or an actual increase in infection rates.

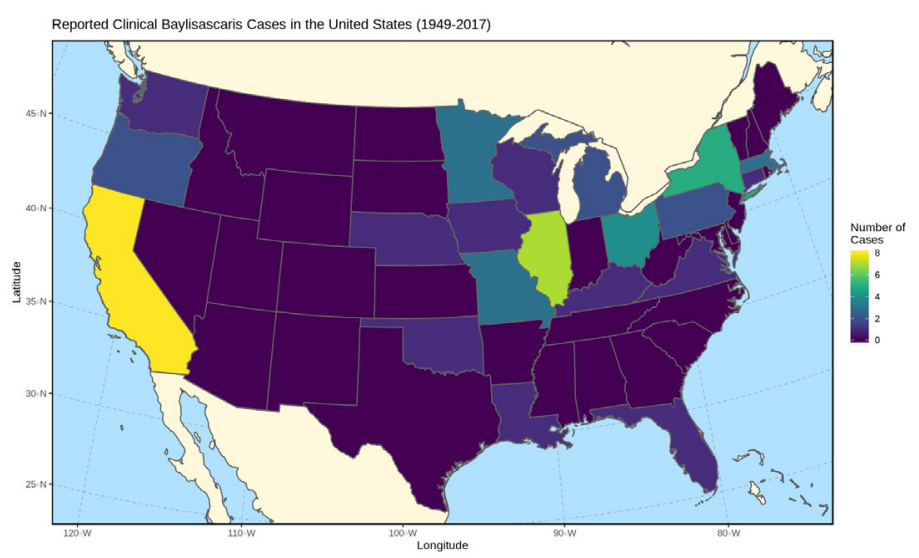

Figure 5. Reported clinical cases of baylisascariasis in the United States (1949-2017), all three presentations (total = 49 cases). Instances where an individual is infected with multiple presentations are counted as one case.

Credits: Data from Kazacos (2016) , Sircar et al. (2016), and Kawakami et al. (2017)

Only identification of larvae is considered confirmatory in diagnosis, but this is not always practical or possible. Baylisascariasis antibodies can be detected in cerebrospinal fluid and serum, and their presence does indicate infection; however, it is not possible to tell if these are from current infection or past exposure. Imaging techniques such as computed tomography (CT) scan and magnetic resonance imaging (MRI) can be used to locate lesions in the brain. A history of exposure to raccoons or their feces is important to diagnosis, as are developmental disabilities, clinical signs specific of each form of baylisascariasis, and conditions such as pica, a psychological disorder causing consumption of non-food items. Refer to the Centers for Disease Control and Prevention (CDC) for more information on baylisascariasis diagnosis. B. procyonis can be confused with Toxocara spp., parasitic roundworms of domestic cats and dogs that can also infect humans. Toxocariasis can cause symptoms similar to those of baylisascariasis, but the disease is normally much less severe, with approximately 13.9 percent of the US population exhibiting antibodies, often with no symptoms. An extensive report on baylisascariasis, including most reported cases, has been published by USGS. 


\section{Asymptomatic and Covert Infection}

Antibodies to B. procyonis have been found in people showing no clinical symptoms. This is most likely due to the individual ingesting a small number of infective eggs, and little to no larvae entering critical areas such as the eyes or the nervous system.

Covert infection occurs when a large enough number of eggs are ingested to cause nonspecific clinical signs, meaning the patient will experience headaches, abdominal pain, a cough, and other symptoms that occur with many other infections. This makes a correct diagnosis very difficult. In both asymptomatic and covert infections, patients have been shown to test positive for antibodies, and would account for a portion of undetected cases.

\section{Neural Larva Migrans}

The most severe form of baylisascariasis is NLM, first described in humans in 1984 (reported cases of NLM in the United States can be seen in Figure 6). An infection with an unknown ascarid larvae in 1973 was later determined to be $B$. procyonis. Most patients with NLM exhibit acute fulminant eosinophilic meningoencephalitis, a sudden and severe inflammation of the membranes that surround the brain and spinal cord. Pica, and geophagia, deliberate consumption of dirt, are important risk factors to baylisascariasis, especially for the NLM form. Developmental disabilities are also a risk factor for NLM. Children are more vulnerable to NLM because of their smaller body size compared to adults, and their comparatively poor hygiene habits, which increase the likelihood that they will consume a large number of larvae. Symptoms of low-level infection include confusion, unsteadiness, learning disabilities, and developmental regression. Symptoms of heavy infection are more severe; including loss of motor skills, impaired vision, sudden lethargy, speech deterioration, stupor, coma, and death.

Treatment is possible, but if it is delayed the patient may survive with severe nerve damage. Only three cases of full recovery from NLM have been reported (with an additional case still under investigation), and these are all relatively recent. Albendazole is the antiparasitic treatment of choice for baylisascariasis and should begin as soon as NLM is suspected. Anti-inflammatory treatments, often corticosteroids, are commonly used as well, because inflammation of the central nervous system plays a critical role in the pathology of baylisascariasis.

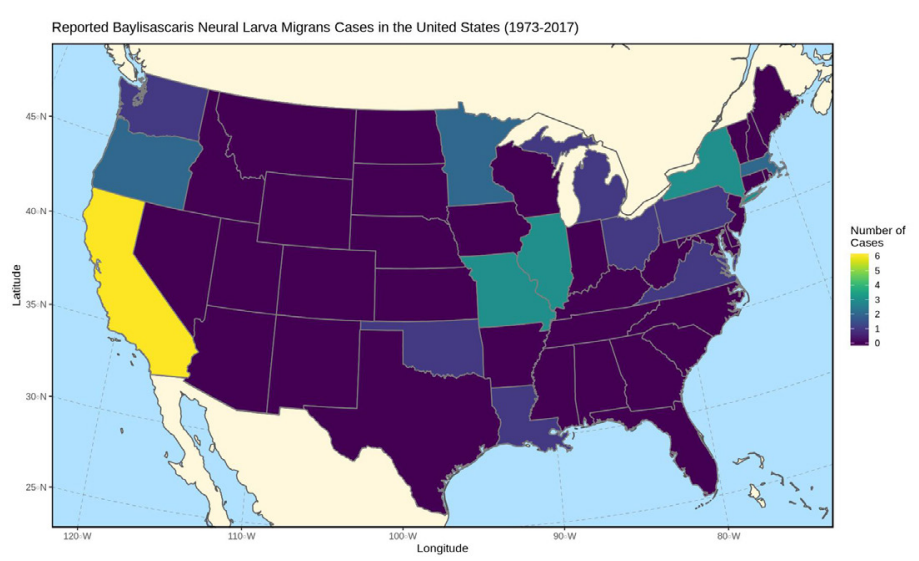

Figure 6. Reported clinical cases of baylisascariasis neural larva migrans in the United States (1973-2017, total = 28 cases). Credits: Kazacos (2016), Sircar et al. (2016), and Kawakami et al. ()

\section{Ocular Larva Migrans}

When B. procyonis invades the eyes it causes OLM. Most cases of OLM occur without NLM or VLM, although they can occur together. The first case was from Florida in 1949, but not classified as B. procyonis until years later (reported cases of OLM in the United States can be seen in Figure 7). In contrast to NLM, OLM is not more common in young children or patients with developmental disabilities. A single larva can enter the eye by chance and cause severe symptoms. Patients with OLM experience sudden blurred vision or vision loss in one eye. Larvae may be visible in the eye. Migration tracks, localized inflammation, and granulomas may be seen. Laser photocoagulation is often used to kill visible larvae. Antiparasitic treatment has been used with mixed results. Corticosteroids are often used to reduce inflammation. OLM by itself is not fatal, but it can cause reduced vision and blindness, which can normally be treated.

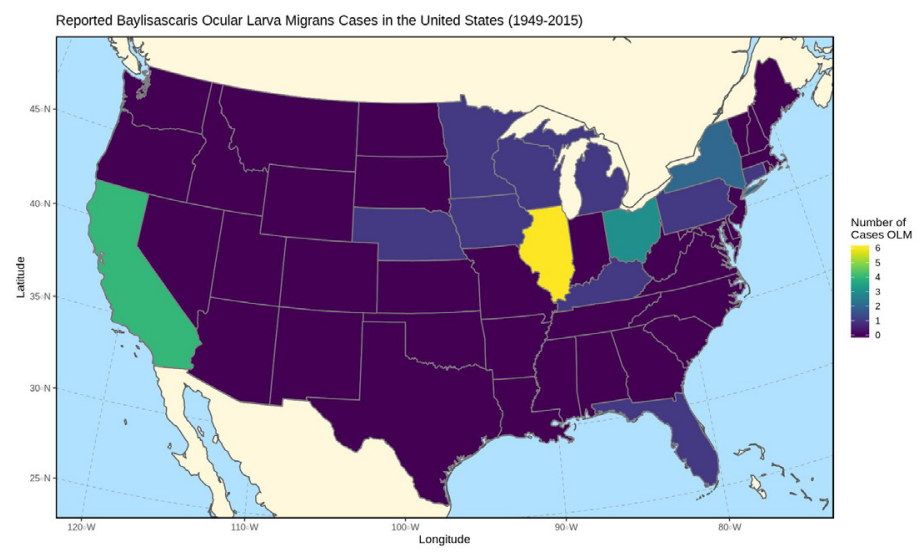

Figure 7. Reported clinical cases of baylisascariasis ocular larva migrans in the United States (1949-2015, total = 24 cases). Credits: Data from Kazacos (2016) and Sircar et al. (2015) 


\section{Visceral Larva Migrans}

VLM occurs when larvae migrate into various internal organs. VLM is the suspected cause of sudden death of a young child in Massachusetts in 1994 who developed a cardiac pseudotumor with several ascarid larvae. This is the only baylisascariasis case where VLM was reported as the possible cause of death. VLM is likely to occur with NLM at a higher rate than is reported, but is overshadowed by the severe neurologic symptoms of NLM.

\section{Baylisascariasis in Wildlife and Pets}

B. procyonis is the most common cause of neural larva migrans in animals, affecting more than 150 known species and accounting for thousands of animal deaths and illnesses in wildlife, zoos, and house pets. Rodents, rabbits, primates, and birds are most susceptible to baylisascariasis.

Domestic dogs are able to act as a definitive host and pass infective eggs without showing symptoms of baylisascariasis, although this is uncommon. At the same time, they can also exhibit fatal NLM. Domestic cats have not been shown to be infected with larvae, even when fed eggs experimentally. Rodents and birds are very susceptible to infection: it can take only a single larva in the brain to prove fatal. Livestock have recently been shown to be infected by $B$. procyonis as well.

Initial signs of baylisascariasis in small mammals include decreased activity, depression, nervousness, rough coat, tremors, head and body tilts, and circling and jumping when disturbed. This can progress into severe head and body tilts, stargazing, rigidity of limbs, weakness, falling over, paddling movements, lack of coordination, blindness, coma, and death. In birds, NLM is characterized by lack of coordination and balance, loss of flight, disorientation, puffed feathers, weakness, tremors, walking in circles, and death.

Removal and prevention of intestinal B. procyonis in dogs can be accomplished with monthly deworming. In addition, veterinarians can look for eggs in fecal samples. Milbemycin oxime (brand name Sentinel), Fenbendazole, pyrantel, moxidectin, and piperazine have shown effectiveness in removing $B$. procyonis in dogs. Information on treatment of NLM in animals is lacking. Antiparasitic and corticosteroid treatments may be used, but prognosis is poor.

\section{Raccoon Feces and Latrines}

Prevention is key, especially for such a disease that is difficult to diagnose and treat. Avoid raccoon feces, and make sure young children and pets do the same. Routine deworming of dogs can prevent them from spreading $B$. procyonis. If using outdoor branches or bark chips in cages and terrariums, bake at a low temperature of $62^{\circ} \mathrm{C} / 144^{\circ} \mathrm{F}$ to kill eggs that may be present. Raccoons defecate in communal sites known as latrines (Figure 8). These areas can be present on bases of trees, branches, woodpiles, fences, tree forks, logs, rocks, roofs, attics, and even in shallow swimming pools. Raccoons have a varied diet, and thus variable feces. They are similar to dog feces, about $3 / 4$ inch in diameter with the tips rounded or broken, two to three inches long, and tubular. The presence of berries is characteristic; feces can also contain crayfish, fish scales, bones, bird seed, and pet food. Bear in mind that eggs can remain infective in the environment long after feces are no longer visible. Evidence suggests that wild animals avoid raccoon latrines, possibly to avoid infection with $B$. procyonis. A recent study showed that raccoons and tolerant rats were attracted to latrines, while more vulnerable animals avoided the areas.

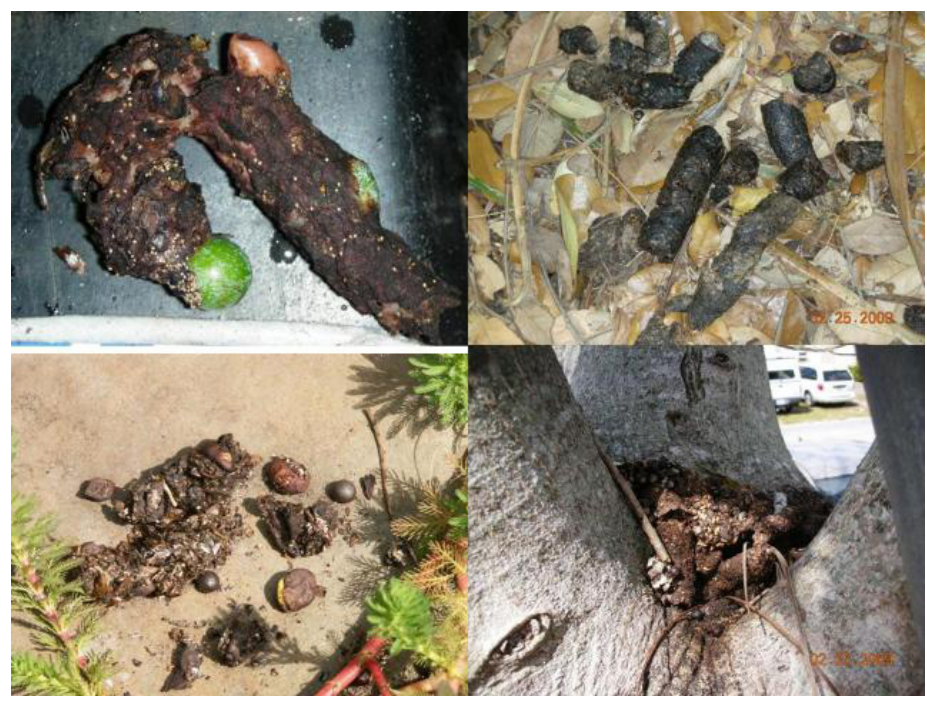

Figure 8. Raccoon feces.

Credits: William Kern, UF/IFAS

It is best to let professionals clean up raccoon latrines, but if you do it yourself, follow CDC guidelines on raccoon latrine removal. Unfortunately, B. procyonis eggs are impossible to kill with commercially available chemicals. Heat will kill eggs instantly, temperatures of $62^{\circ} \mathrm{C} / 144^{\circ} \mathrm{F}$ completely deactivate eggs in under one minute as shown by the CDC. Use boiling water and flame safely to decontaminate latrines. Contact your local fire department for guidelines when using fire. Any contaminated materials 
that are disposable, such as attic insulation, should simply be replaced. Always wear protective gloves, goggles, and masks; and wash clothes in hot water after exposure to raccoon feces.

\section{Conclusions}

With increased development and habitat fragmentation, the risk that humans will come into contact with raccoons and other adaptable species increases. Raccoons readily adapt to urban environments (Figure 9), thus increasing the chance for raccoon-borne diseases to spread to humans and pets. There are a few simple solutions to prevent the spread of raccoon-borne diseases. First, never feed wild animals. In addition to being illegal, feeding wildlife causes animals to associate humans with food, and sometimes to become dependent upon humans. They may lose their natural fear, and they may become aggressive. Feeding wildlife also increases population density and territorial overlap, and the chances of disease transmission between individuals. In addition to not feeding wildlife, feed pets inside, or at least bring food in at night. Purchase raccoon-proof garbage cans if they are getting into your garbage. If you have an unwanted raccoon on your property, call a wildlife removal specialist. The Florida Fish and Wildlife Conservation Commission keeps a list of wildlife removal specialists on their website. Removing a raccoon without professional assistance is difficult and risky, and there is a very high chance the raccoon will come back, or that you remove only part of a family and leave orphans.

Raccoon-borne diseases pose a significant threat to humans, wildlife, zoo animals, livestock, and pets. With knowledge and simple measures, we can avoid these hazards and be proactive in treatment. Most problems arise when people attract raccoons intentionally or unintentionally. When treated with respect and caution, raccoons can live near humans as an interesting part of the urban ecosystem.

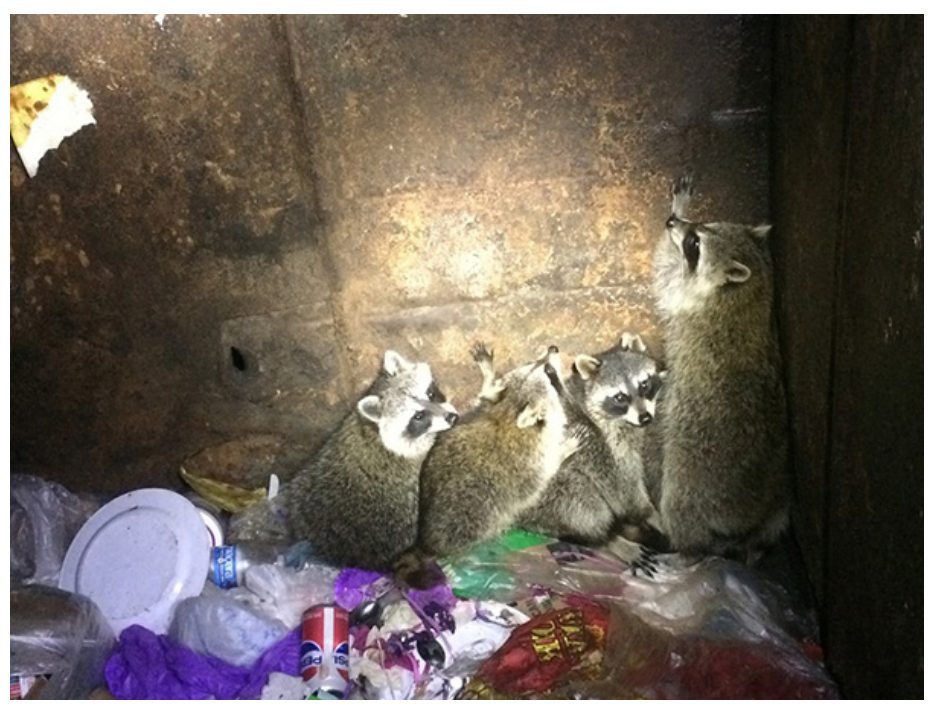

Figure 9. A group (gaze) of five raccoons in a dumpster, a common gathering spot.

Credits: Caitlin Jarvis

\section{Sources and Further Reading}

Blizzard, E. L., M. J. Yabsley, M. F. Beck, and S. Harsch. 2010. "Geographic Expansion of Baylisascaris procyonis Roundworms, Florida, USA.” Emerging Infectious Diseases 16:1803-1804. https://doi.org/10.3201/eid1611.100549

Centers for Disease Control and Prevention. 2011. "Raccoon latrines: identification and clean-up." https://www.cdc. gov/parasites/baylisascaris/resources/raccoonlatrines.pdf

Centers for Disease Control and Prevention. 2013. “Toxocariasis." https://www.cdc.gov/parasites/toxocariasis/

Gavin, P. J., K. R. Kazacos, and S. T. Shulman. 2005. "Baylisascariasis.” Clinical Microbiology Reviews 18:703-718. https://doi.org/10.1128/CMR.18.4.703-718.2005

Graeff-Teixeira, C., A. L. Morassutti, and K. R. Kazacos. 2016. "Update on Baylisascariasis, a Highly Pathogenic Zoonotic Infection." Clinical Microbiology Reviews 29:375399. https://doi.org/10.1128/CMR.00044-15

Kawakami, V., A. Casto, N. Natarajan, A. Snyder, J. Mosser, J. Bonwitt, M. P. Kronman, and M. Kay. 2018. "Notes from the Field: Baylisascaris procyonis Encephalomyelitis in a Toddler - King County, Washington, 2017." Morbidity and Mortality Weekly Report 67:79-80. https://doi.org/10.15585/ mmwr.mm6702a6

Kazacos, K. R. 2016. “Baylisascaris Larva Migrans.” U.S. Geological Survey Circular 1412. https://doi.org/10.3133/ cir1412 
Sapp, S. G. H., L. N. Rascoe, P. P. Wilkins, S. Handali, E. B. Gray, M. L. Eberhard, D. M. Woodhall, et al. 2016.

"Baylisascaris procyonis Roundworm Seroprevalence among Wildlife Rehabilitators, United States and Canada, 2012-2015." Emerging Infectious Diseases 22:2128-2131. https://doi.org/10.3201/eid2212.160467

Sircar, A. D., F. Abanyie, D. Blumberg, P. Chin-Hong, K. S. Coulter, D. Cunningham, W. C. Huskins, et al. 2016. "Raccoon Roundworm Infection Associated with Central Nervous System Disease and Ocular Disease-Six States, 2013-2015." Morbidity and Mortality Mortal Weekly Report 65:930-933. https://doi.org/10.15585/mmwr.mm6535a2

Weinstein, S. B., C. W. Moura, J. F. Mendez, and K. D. Lafferty. 2017. "Fear of feces? Tradeoffs between disease risk and foraging drive animal activity around raccoon latrines." Oikos 127:927-934. https://doi.org/10.1111/oik.04866 